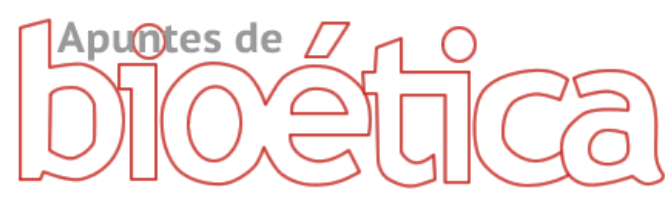

https://doi.org/10.35383/apuntes.v2i1.241

\title{
Resolución de conflictos en familias con hijos adolescentes
}

\author{
Fabiola Guerrero San Martín ${ }^{1}$
}

\section{INFORMACIÓN DEL ARTÍCULO RESUMEN}

Historia del artículo:

Recibido el 20 de marzo de 2019

Aceptado el 20 de julio de 2019

Los conflictos, siendo una situación recurrente y cotidiana, se dan como

Palabras claves:

Familia

Adolescentes

Resolución de conflictos

Estrategias

resultado de la convivencia e interrelación de las personas. La resolución de conflictos dentro de las familias con hijos adolescentes busca identificar los conflictos existentes, diferenciando diversas estrategias de resolución dirigidas a sus integrantes. El presente artículo presenta una revisión bibliográfica, descriptiva y teórica de estudios relacionados a los conflictos, su manejo y su relación en las familias con hijos adolescentes, basados en la Teoría Sistémica Familiar, de aportes de varios autores y de manera significativa las directivas de la metodología Harvard para la negociación, presentando pautas y estrategias que ayuden eficaz y eficientemente a la resolución de conflictos, buscando abrir una puerta de ayuda y prevención con aportes significativos para su consideración y aplicación dentro de las familias, en especial donde hay hijos adolescentes.

\section{Resolution of conflict in families with adolescent children}

\section{ABSTRACT}

Keywords:

Family

Teenagers

Conflict resolution

Strategies
Conflicts, being a recurring and daily situation, occur as a result of the coexistence and interrelation of people. The resolution of conflicts within families with adolescent children seeks to identify existing conflicts, differentiating various resolution strategies aimed at their members. This article presents a bibliographic, descriptive and theoretical review of studies related to conflicts, their management and their relationship in families with teenage children, based on the Family Systemic Theory, of contributions from several authors and in a significant way the directives of the Harvard methodology for negotiation, presenting guidelines and strategies that effectively and efficiently help to resolve conflicts, seeking to open a door

\footnotetext{
${ }^{1}$ Maestro en Persona, Matrimonio y Familia, Licenciada en Psicología humana, realiza consultoría y asesoría en área de matrimonio y familia, noviazgo, relaciones interpersonales, caracterología. Lima, Perú. Email: f.querrerosanmartin@gmail.com. ORCID: https://orcid.org/0000-0002-8490-6808
} 
for help and prevention with significant contributions for consideration and application within families, especially where there are teenage children.

\section{Introducción}

En el ejercicio de la práctica profesional, en la cercanía y el acompañamiento realizado con familias de adolescentes, se perciben y describen las situaciones de conflicto, así como la forma en que estos son tratados o ignorados al interior del hogar. Esto sumado a los diversos estudios realizados sobre realidades familiares nacionales ${ }^{2}$ e internacionales ${ }^{3}$ llevó a observar los diversos cambios que se dan en las estructuras familiares (UNICEF, 2003) y de acuerdo a estos contextos y realidades, plantear la búsqueda de las mejores estrategias de resolución de conflictos al interior de las familias.

Esta realidad se repite constantemente haciéndose más recurrente la problemática en los hogares, manifestándose en: separación, abandono, violencia y desinterés entre los integrantes de una misma familia, evidenciándose entre los hijos, inadecuadas

\footnotetext{
2 Siendo los tomados como referencia los de Graza Evaristo, Shirley (2012) con su tesis "Relación entre funcionalidad familiar y nivel de violencia escolar en los adolescentes de la Institución educativa Francisco Bolognesi Cervantes $N^{\circ} 2053$ Independencia 2012" en donde se recalca la importancia de la funcionalidad familiar para hacer frente a situaciones de riesgo en los adolescentes. Igualmente los aportes de Chuquimajo Huamantumba, Silvia (2014) con su tesis "Personalidad y clima social familiar en adolescentes de familia nuclear biparental y monoparental" en donde se resalta la importancia de cimentar la personalidad de los adolescentes dentro de un contexto familiar diferenciado en el cual es propicio el entender los cambios propios de su estructura y organización.

3 Diversos estudios dados a nivel internacional que sustentan esta investigación, corroboran datos de percepción de clima familiar, conflictos en el hogar, bajo rendimiento y adicciones, entre los que destaca Feixas I Viaplana, Guillem; Muñoz Cano, Damaris; Compañ Felipe, Victoria y Montesano Del Campo, Adrián (2012) con su aporte teórico brindado por medio de la Facultad de Psicología de la Universidad de Barcelona, titulado "El modelo Sistémico en la intervención familiar" por el cual se sitúa el modelo sistémico de intervención psicológica junto a la terapia familiar como un formato de intervención estructurado, definido por sus funciones, retroalimentación y relaciones de interdependencia que lleva a entender a la familia como un todo organizado. Igualmente, la tesis "Narrativas sobre la familia en adolescentes pertenecientes a familias ensambladas" de Allen Peralta, Silvia (2012) brinda como aporte el ampliar la mirada de la percepción de las realidades familiares a partir de sus historias personales de impacto en el desarrollo del adolescente.
}

relaciones interpersonales, estados depresivos (Arenas Ayllón, 2009), soledad, baja autoestima, poca tolerancia, egoísmo, llegando en algunos casos a estados agresivos (Matalinares, y otros), adicciones y rebeldía entre otros, que indudablemente afectan también su rendimiento académico (García, Sánchez, \& Robles, 2009) y que traen consigo el deterioro y la paulatina desintegración familiar.

Frente a esta problemática y pese a contar con estudios y técnicas profesionales de abordaje recomendadas a las familias, encontramos que en muchos casos la indiferencia para buscar acceder a estas ayudas o las desfavorables condiciones económicas de las familias impiden se concrete esta recomendación. Surge así la búsqueda por brindar propuestas de mejora y nuevas pautas de ayuda para resolver conflictos dentro de las familias donde encontramos adolescentes.

Surge así la inquietud por responder a esta necesidad $y$ hacer frente $a$ esta problemática, presentando como objetivo principal el brindar propuestas de mejora $y$ pautas de ayuda por medio de estrategias para la resolución de conflictos en familias con hijos adolescentes, y como objetivos específicos: identificar los conflictos en las familias, diferenciar estrategias para la resolución de conflictos y especificar estrategias de resolución de conflictos basadas en el método de negociación Harvard.

Esta propuesta lleva a identificar primeramente los conflictos existentes en las familias; diferenciando las diversas estrategias dirigidas a ellas para el mejor manejo de los conflictos al interior del hogar; y precisando 
estrategias de resolución de conflictos considerando los aspectos que las diferencian.

Cumpliendo estas pautas, se realizó la revisión teórica ${ }^{4}$ basada en la Teoría Sistémica Familiar, que aunada a experiencias sobre dinámica familiar y sobre la resolución de conflictos basada en el modelo Harvard ${ }^{5}$ de negociación, contribuyen al manejo adecuado de los conflictos con el empleo de los siete elementos propios de este modelo, aportando con esto, una guía eficaz frente a situaciones conflictivas, considerando las estrategias más adecuadas para su resolución y realizando así una oportuna prevención tanto en los padres de familia como en los hijos.

Con este aporte en estrategias y de lo que de él se desprende, se espera brindar una ayuda consistente y adecuada para ser utilizada en las familias de estudiantes adolescentes, una contribución para aceptar, afrontar y aprender de cada situación de conflicto acaecida en el hogar, buscando que esta lleve a sus integrantes a madurar y a crecer en unidad como familia.

\section{Metodología}

Esta ha sido cualitativa, básica y descriptiva. Este enfoque, referido a la investigación usada principalmente en las ciencias sociales, buscó identificar la naturaleza del problema a tratar y describir los diferentes aspectos que corresponden al contenido del

\footnotetext{
${ }^{4}$ De acuerdo a diversos autores que sirven como soporte para este estudio, destacan los estudios de la Teórica Sistémica familiar con Salvador Minuchin como autor principal, Jay Haley y otros, el Proyecto Harvard de negociación propuesto por Roger Fisher, el estudio de la teoría de resolución de conflictos con A.J.R. Gromm y los aportes de investigaciones precedentes entre las que destaca el Instituto Complutense de Mediación y gestión de conflictos, así como el sustento espiritual para fortalecer estos planteamientos, encontrados en el catecismo de la Iglesia Católica, los documentos eclesiales sobre familia y los escritos de San Juan Pablo II así como los recientes aportes del actual Papa Francisco.

${ }^{5}$ Modelo propuesto en el año 1981 por los profesores universitarios Roger Fisher, William Ury y Bruce Patton en su libro "Obtenga el Si" referido al Proyecto de negociación Harvard que plantea la búsqueda de soluciones basadas en la negociación desde la aplicación de siete elementos que forman parte de este proceso. Así mismo, se ve reforzado este planteamiento desde el aporte brindado en el Workshop de negociación. CMI Internacional Group. Cambridge. 1998, siendo este es un grupo de trabajo vigente y base para el tratamiento de la resolución de conflictos.
}

tema del estudio. Por ella se realizó una búsqueda teórica y bibliográfica de los aspectos particulares del tema, mediante el análisis documental, caracterizando y describiendo los contenidos -materia de estudio- exponiendo también las teorías que sustentan este artículo.

Los criterios éticos utilizados han considerado lo establecido en el Código de niños y adolescentes promovido por el Ministerio de la Mujer y Poblaciones Vulnerables y sustentado en la Comisión Internacional sobre los derechos del Niño (CIDN) y por la UNICEF, así como la Comisión para la protección de las personas objeto de la experimentación Biomédica y de la Conducta. Estos valoran:

- La importancia y respeto de la persona humana, en su derecho fundamental de ser tratado como persona y gozar de derechos que signifiquen soporte y desarrollo, en su alimentación y vivienda, y en su formación integral y educación que le permita una personalidad segura, con actitudes positivas hacia el mundo.

- La búsqueda del bien común, la justicia y la paz social, salvaguardando la dignidad de las personas y procurando la equidad tanto en derechos como en deberes, contribuyendo así a la defensa de la vida y la recuperación de la dignidad humana, en la verdad y la justicia .

- La armonía en la familia, en la importancia que tiene en la sociedad, en el mundo, en cada persona. Es por ella que es posible guiar, orientar, formar, enseñar valores, ayudar a relacionarse con los demás, a ser soporte emocional y afectivo para sus miembros.

\section{Resultados, análisis y discusión}

\section{EL CONFLICTO EN LAS FAMILIAS}

La familia, es y será siempre un tema motivo de alegría y esperanza, así como de preocupación y análisis. Es en ella que se forma la persona y de ella es que nace la vocación para 
el ser y para el buen hacer. La vocación a la vida, al servicio, al matrimonio, a la realización humana en la paternidad y maternidad se fundamentan en la familia ${ }^{6}$. Por ello, dentro de su ciclo vital $^{7}$ no es de sorprender que atraviese por diversas fases de equilibrio y desequilibrio que afecten la homeostasis del hogar, entendiéndose estos cambios como parte de su desarrollo.

El conflicto es parte de estas fases. Este se origina por la convivencia y las relaciones humanas de manera cotidiana y puede surgir en cualquier situación en donde hayan diversos modos de pensar u obrar, en los intercambios de opiniones, en los diversos niveles de comportamiento que pueden llegar a la intolerancia, discusión y enfrentamientos. Sin embargo es necesario especificar, al referimos al término conflicto, que muchas veces este puede entenderse dentro de un contexto orientado a temáticas de negociación o situaciones puramente negativas, sin ser conscientes de que al existir los conflictos desde siempre, estos deben ser abordados oportunamente para evitar percepciones erradas que conlleven a situaciones negativas dentro de la familia.

Es así que podemos encontrar diversas definiciones en lo referente al significado del conflicto a partir de su etimología (Real Academia de la lengua española) con términos (Fuquen Alvarado, 2003) entre los que encontramos: chocar, afligir, infringir, confrontación, problema, dificultad, pelea,

\footnotetext{
6 "[...] La familia posee vínculos vitales y orgánicos con la sociedad, porque constituye su fundamento y alimento continuo mediante su función de servicio a la vida. En efecto, de la familia nacen los ciudadanos, y estos encuentran en ella la primera escuela de esas virtudes sociales, que son el alma de la vida y del desarrollo de la sociedad misma. Así la familia, en virtud de su naturaleza y vocación, lejos de encerrarse en sí misma, se abre a las demás familias y a la sociedad, asumiendo su función social". JUAN PABLO II, Familiaris Consortio $\mathrm{N}^{\circ} 42$.

7 "[...] implica entender el desarrollo como un proceso que dura toda la vida, abarcando tanto aspectos continuos como discontinuos, en el que las personas se adaptan mediante mecanismos de optimización selectiva, en una dinámica de ganancias y pérdidas, con cambios asociados a la edad y a la plasticidad individual y bajo la influencia de múltiples factores contextuales. BALTES, STAUNDINGER y LINDERBERGER, 1999, citados por (Alonso Struyck, 2004)
}

desgracia, violencia, oposición, mala suerte, materia de discusión, entre otros.

Esta terminología, en el contexto de familia, al tener características de frecuencia y relación ha sido definida operacionalmente tomando en cuenta los criterios y aportes de diversos autores, estudios y aportes en el campo del manejo de conflictos y del ámbito familiar, de la siguiente manera:

"El conflicto en la familia es el proceso vinculante $y$ de interrelación, originado por la forma en que se produce la convivencia misma, por los estilos de comunicación y la interacción en las relaciones humanas que se dan entre sus miembros y en donde cada parte aporta su particular percepción de la realidad de la cual es partícipe y en donde busca imponer sus motivaciones, puntos de vista, necesidades e incluso sus gustos $y$ preferencias, generando discrepancia y ruptura en el diálogo familiar. Este proceso, sin embargo, cumple al mismo tiempo un rol adaptativo en el funcionamiento familiar $y$ en el desarrollo de todos sus integrantes, facilitando -al ser bien abordada- la adquisición de habilidades para tolerar y resolver las situaciones difíciles que se presenten manteniendo estable la relación familiar con adecuadas herramientas de resolución"s.

Dentro del ciclo vital ${ }^{9}$ de la familia, no es de sorprender que se atraviese por diversas fases de equilibrio y desequilibrio que afecten la

\footnotetext{
8 Definición operacional, propuesta de la autora, basada en la teoría sistémica, aportes de Minuchín y Fisher, así como investigaciones precedentes de Allen Peralta (2012), Alonso Struick (2004), Burgos Gaibor (2012), Graza Evaristo (2013), Chuquimajo Huamantumba (2014) Feixas y otros (2012), Cid-Monckton y Pedrão (2011), Motrico, Fuentes y Bersabé (2001), y otros autores citados en este estudio.

9 " [...] implica entender el desarrollo como un proceso que dura toda la vida, abarcando tanto aspectos continuos como discontinuos, en el que las personas se adaptan mediante mecanismos de optimización selectiva, en una dinámica de ganancias y pérdidas, con cambios asociados a la edad y a la plasticidad individual y bajo la influencia de múltiples factores contextuales. Baltes, Staundinger y Linderberger (1999), citados por Alonso Struyck (2004, pág.14)
} 
homeostasis del hogar, esto es parte de su desarrollo, sin embargo la manera como la familia afronta estas etapas hacen de ella una familia en vías de madurez.

Es así que tendremos dentro de las fases de este ciclo evolutivo aquel considerado como Familia con hijos adolescentes ${ }^{10} \mathrm{o}$ en edad escolar que es en el que ubicamos a nuestro tipo de familia estudiada. Durante esta etapa se forman rituales de socialización para los hijos y aquí se hace necesario redefinir el acompañamiento de los padres hacia sus hijos considerando los cambios físicos y psicológicos que presentan ambas partes, pues mientras los hijos se enfrentan a la adolescencia, los padres tendrán la tendencia a ser cuidadores de sus propios padres.

Es importante mencionar que las etapas del ciclo evolutivo familiar (Alonso Struyck, 2004, pág. 53) son ajustables a cada familia, más aun teniendo en cuenta que existen muchas variedades de convivencia familiar cada vez menos tradicionales, considerando también aquellas que atraviesan situaciones de divorcio, separación, enfermedad, duelo, adopción e incluso su reconstitución.

Esta etapa de desarrollo familiar es tendente a ser difícil para la familia debido a que los padres atraviesan al mismo tiempo diversas crisis como la llamada crisis de la mediana edad, el saber o proyectar que sus hijos adolescentes en un futuro no muy lejano abandonarán el hogar, y el saber que sus propios padres atraviesan el ciclo de la vejez, por lo que tienen que hacer frente a tensiones que vienen de tres fuentes: los hijos, los padres y su propio ciclo vital (Ortiz Granja, 2008, pág.231). En esta etapa, la familia tiene que renegociar las reglas de la relación familiar y los padres tienen que empezar a pensar en lo que sucederá cuando se queden nuevamente solos.

\footnotetext{
${ }^{10}$ Para esta etapa en particular, vemos que "Las familias con adolescentes, se encuentran ante un entramado de cambios y mecanismos de adaptación a los mismos". Alonso Struyck (2004, pág. 52)
}

Son diversos los cambios que atraviesan las familias durante esta etapa, sin embargo es importante resaltar que el análisis y estudio de los conflictos en las familias, producidos entre padres e hijos durante la adolescencia ${ }^{11}$, no debe buscar hacer de este un problema mayor en esta etapa, por lo contrario, debe entenderse como una situación natural que se da en una relación y que tiene un matiz íntimo (Motrico, Fuentes , \& Bersabé , 2001, pág. 2). Por ello es importante considerar que "el conflicto se hace anormal solamente cuando se convierte en norma, o sea cuando caracteriza el conjunto del comportamiento" (Rodríguez Estrada, 1989, pág. 2) por lo cual es importante abordar los conflictos, siendo conversados y resueltos para el mejor desarrollo de las relaciones familiares, de otra manera nos encontraríamos con una realidad llena de conflictos más profundos aún, por la incapacidad o permisividad para asumirlos y buscar soluciones oportunas.

\section{ASPECTOS DIFERENCIALES DE LAS FAMILIAS}

Para comprender de mejor manera la existencia y abordaje de los conflictos en las familias, es importante tener en cuenta que cada familia es única y diferente, por ello, pese a tener similitudes, esta debe ser entendida considerando las premisas de la Teoría general de sistemas- como un sistema dentro de otros sistemas $^{12}$. Eso es algo que se debe resaltar, destacar y valorar para realizar una reflexión sobre las diversas situaciones particulares (Maganto) que estas atraviesan y que se ven reflejadas en la forma como se identifican las singularidades que poseen.

\footnotetext{
11 La etapa de las familias con hijos adolescentes, se verá inmersa en los cambios de adaptación, que, a decir de estudiosos como Carter (1989) y Buelga (1993) se dará en las diversas etapas el ciclo vital y en donde al aparecer las crisis, estas podrán favorecer el equilibrio en las familias o por lo contrario, estancar su desarrollo. (Alonso Struyck, 2004, pág. 52)

12 De acuerdo a las premisas de la TGS expuestos en el marco teórico el Capítulo I referido al preámbulo de la Teoría sistémica familiar con la concepción de la TGS dentro de la estructura familiar. (Espinal, Gimeno Collado, \& González Sala, 2004, pág. 3).
} 
Son diversas y variadas, sin embargo las particularidades de las familias se han ordenado y dispuesto considerando la clasificación familiar planteada por Minuchín, consideradas por Ortiz y valoradas por el Instituto Complutense de Mediación y gestión de conflictos. Encontramos así:

- Familias en donde alguno de los miembros atraviesan problemas extrafamiliares, con dificultades o problemas laborales;

- Familias que viven una experiencia de estrés extrafamiliar;

- Familias con estrés por momentos evolutivos naturales de la familia o por cambios significativos de la misma;

- Familias con problemas físicos o psicológicos de algún miembro que obliga a adoptar medidas de funcionamiento no habituales;

Estas particularidades requieren una dedicación especial de tiempo y afecto, que involucra a toda la familia y en donde el riesgo está en el desgaste que se pueda dar entre sus integrantes.

Consideramos también como una diferenciación de las Familias, aquellas basadas en las categorías de Minuchín, Ortiz y otros investigadores entre las que encontramos:

- Familias nucleares ${ }^{13}$,

- Familias extensas o extendidas,

- Familias monoparentales ${ }^{14}$,

- Familias de tres generaciones ${ }^{15} y$

- Familias reconstituidas, reconstruidas 0 simultáneas ${ }^{16}$.

\footnotetext{
${ }^{13}$ En este tipo de familia, solo encontramos al padre, madre e hijos de la relación. (Ortiz Granja, 2008, pág. 200)

14 "aquella en la cual el padre o la madre han dejado la familia por diversas circunstancias como muerte, viaje, separación, etc., y en la que el otro miembro de la pareja se queda a cargo de los niños" (Ortiz Granja, 2008, pág. 200).

${ }^{15}$ Se trata de una familia en la cual viven miembros de la tercera generación, como es el caso de un abuelo o abuela o ambos, de cualquier rama de la familia. (Ortiz Granja, 2008, pág. 200)

${ }^{16}$ Aparece cuando una familia nuclear sufre la ruptura de la pareja y el hombre o la mujer vuelven a formar pareja, algunas veces llevando sus propios hijos a la relación por lo que se dan casos en los que en la nueva familia viven, por ejemplo, el hombre y los hijos de su anterior relación, la mujer y los hijos de su anterior relación y los hijos que nacen en esta nueva
}

Entender estas diferencias significará ampliar la visión que se tiene sobre el conflicto mismo y la percepción que las familias pueden tener de este, al identificarlo en su propia realidad.

\section{PERCEPCIÓN E IDENTIFICACIÓN DEL CONFLICTO EN LAS FAMILIAS CON HIJOS ADOLESCENTES}

Cuando los miembros de una familia perciben en su hogar, una situación de conflicto, lo pueden interiorizar y/o exteriorizar de diversas formas pero siempre en relación a sus integrantes. Recordemos que la familia es un sistema que a su vez se compone de otros subsistemas (Gonzales Gallegos, 2007, pág. 111) para poder interactuar. El subsistema conyugal, entre esposos es uno de ellos, otro es el subsistema parental cuyos integrantes son los mismos que el anterior y que se constituyen con la llegada del primer hijo, y el subsistema filial o fraterno constituido propiamente por los hijos, los cuales constituyen un núcleo diferenciado de miembros del sistema y al mismo tiempo se encuentran vinculados de manera específica. Aquí es donde se dan las relaciones particulares entre sus miembros. ${ }^{17}$ Es por ello que "las relaciones en el seno de la familia entrañan una afinidad de sentimientos, afectos e intereses que provienen sobre todo del mutuo respeto de las personas $[\ldots]^{\prime 18}$ de tal manera que estas relaciones pueden ser positivas como negativas, dependiendo en gran parte de la percepción de sus integrantes.

Los estudios realizados en familias con hijos adolescentes, analizados por investigaciones y teorías sistémicas, confirman la existencia de diversos conflictos comunes a ser clasificados y enumerados, los mismos que ayudan a contextualizar la situación familiar

relación (coloquialmente: "los míos, los tuyos y los nuestros"). (Ortiz Granja, 2008, pág. 201)

${ }^{17}$ Existen también otros subsistemas relacionados con las familias, como el de Autonomía, de cuidados mutuos, afectivo-empático, de definición de límites intergeneracionales, de resolución de conflictos, de hermanos, sensorial-sexual y comunicacional. (Gonzales Gallegos, 2007, pág. 112)

${ }^{18}$ CATECISMO DE LA IGLESIA CATÓLICA N²206 
existente en el hogar ${ }^{19}$. Encontramos así que los podemos clasificar como aquello que es:

- Propio de las opciones y costumbres sociales (elección de amistades y; enamoramiento; empleo del tiempo libre, horarios de salida y retorno de casa, vestimenta, peinado y forma de expresarse, entre otros);

- Propio de las responsabilidades (cumplimiento de deberes y cumplimiento de responsabilidades familiares; administración del dinero, cuidado de sus pertenencias, aseo de la habitación personal, control de medios de comunicación, internet, redes, celular, TV, videojuegos, etc.);

- Propio de los estudios (rendimiento académico; calificaciones; cumplimiento de tareas en casa y colegio; hábitos de estudio, asistencia a clases, conducta y actitud en el centro de estudios y con profesores);

- Propio de las relaciones familiares: (frecuencia en la comunicación; respeto entre padres e hijos; relaciones fraternas entre los miembros, discusiones, celos, peleas entre hermanos, la autonomía frente a la familia, relaciones de autoridad en casa con los abuelos u otros familiares, afectación y adaptación frente a la separación de los padres $o$ ante una nueva relación que pudieran tener, mudanza, fallecimiento de algunos de los miembros, etc.);

- Propio de los valores morales (conductas perjudiciales: Beber, fumar o consumir drogas; el tipo de lenguaje utilizado al hablar, conducta sexual de acuerdo a su edad; el cumplimiento de las leyes, la participación en actos religiosos o cívicos, etc.).

La percepción que cada integrante pueda tener de uno o varios conflictos al interior de la familia, difiere una de otra, de acuerdo a su realidad particular, y tendrá influencia en la manera como se puedan plantear las estrategias

\footnotetext{
${ }^{19}$ Según aportes de temas de conflicto comunes a las familias de estudiantes adolescentes y estudios citados por diversos autores referidos a los conflictos en las familias. BOSMA et al.,1996; COLLINS Y LAURSEN, 1992; LAURSEN, COY Y COLLINS, 1998; NOLLER, 1994), citados por (Motrico, Fuentes , \& Bersabé , 2001, pág. 3)
}

de resolución de conflictos frente a ella, por ello se hace necesario poder hacer la diferenciación de las mismas para buscar las mejores alternativas de solución.

\section{DIFERENCIANDO ESTRATEGIAS PARA LA RESOLUCIÓN DE CONFLICTOS EN LAS FAMILIAS}

La Teoría Sistémica Familiar Basada en la Teoría general de sistemas ${ }^{20}$ permite analizar que cada familia, reconocida como un sistema, es decir una unidad, se encuentra dentro de una red más amplia y en relación con otros sistemas. Por ello, generar estrategias desde la TSF significa evidenciar que esta teoría es la que aborda la realidad familiar, su contexto y comprensión con mejores perspectivas de resultados frente a la resolución de conflictos. Por ellos el modelo sistémico se ha expandido durante los últimos años y es aceptado actualmente en diversos ámbitos demostrando ser eficaz y flexible al tratar diversos problemas (Feixas I Viaplana, Muñoz Cano, Compañ Felipe, \& Montesano del Campo, 2012, pág. 4), más aún en las familias ${ }^{21}$, consideradas como sistema, "al que le son aplicables los principios propios de los Sistemas Generales, y también otras características relevantes, que facilitan su comprensión y que son específicas de los sistemas familiares", contribuyendo así en gran forma a los procesos familiares así como a su evaluación e intervención.

Considerándolo también como un gran aporte para el fortalecimiento de las familias, tenemos los apuntes de la Exhortación apostólica sobre la familia, Amoris Laetitia (Papa Francisco, 2016, págs. 113-119) del Papa Francisco en donde se menciona repetidamente

\footnotetext{
${ }^{20}$ La Teoría General de los Sistemas surge desde la formulación que hiciera Von Bertalanffy en 1968 buscando dar explicación a los principios sobre la organización de muchos fenómenos naturales y que en la actualidad es aplicada al conocimiento de muchas otras realidades, tanto naturales como ecológicas, medioambientales, sociales, pedagógicas, psicológicas o tecnológicas. HERNÁNDEZ, 1989, 1993 y 1997; CASTILLEJO Y COLOM, 1987; CUSINATO, 1992; CAMPANINI Y LUPPI, 1996; RODRÍGUEZ DELGADO, 1997, citados por (Espinal, Gimeno Collado, \& González Sala, 2004, pág. 1)

${ }^{21}$ Al considerar a la familia como sistema, se espera de ella ser una fuente de salud social ejerciendo adecuadamente sus funciones básicas. (Maganto, pág. 12)
} 
la necesidad de fortalecer y dar el lugar que corresponde a la familia, tan golpeada en estos últimos tiempos y en donde reside la fuente de la vida y en donde cada miembro es considerado parte importante de este engranaje en la cual hallamos la ampliación de la llamada, familia grande, donde se hace necesaria la participación y la presencia de todos para un bien mayor hacia la sociedad, y en donde son inevitables las situaciones de crisis de las cuales se obtiene el aprendizaje necesario que implica superar obstáculos y afinar el oído del corazón (Papa Francisco, 2016, pág. 140) entre sus miembros.

Cuando se utilizan estrategias adecuadas para resolver los conflictos, estos pueden contribuir positivamente a la madurez y mejor desarrollo del adolescente, a mejorar el funcionamiento familiar y las relaciones padremadre-hijo-hija, así como afinar la comprensión y el reajuste familiar en las diversas etapas de desarrollo, más aún con la llegada a la adolescencia. (Bernedo Muñoz, Fuentes Rebollo, \& Fernandez Molina, 2005, pág. 371)

(Rodríguez Estrada, 1989) presenta ciertas técnicas -estrategias- para manejar conflictos. Estos marcan una pauta interesante para la ejecución de los siete pasos de negociación Harvard en el tratamiento de los conflictos familiares, los cuales encontramos entendible, comprensible y viable, y con los cuales mostramos coincidencias para la estructuración de estrategias basadas en principios en consonancia con la TSF.

- Considerar el análisis de problemas formulando preguntas para diagnosticar el problema y analizar cómo se está manejando la situación; utilizando un lenguaje positivo evitando expresiones contrarias hacia las personas (Rodríguez Estrada, 1989, pág. 50).

- Desarrollar en sí mismo y en los demás integrantes de las familias, habilidades de negociación: hablar sin atacar, escuchar con empatía, facilitar la cooperación poniendo énfasis en los valores comunes.
- Tener la capacidad de decidir y actuar cuando sea necesario, buscando llegar a un acuerdo unánime aunque en varios casos signifique poner los asuntos a votación.

- De ser necesario, recurrir a la mediación y arbitraje de personas respetadas por las partes en conflicto (legitimidad).

A este aporte, podemos agregar aquellos brindados por el Instituto Complutense de mediación y gestión de conflictos (García Villaluenga, y otros, 2010) que contribuyen a enriquecer estas estrategias:

- Mostrarse abiertos al diálogo, buscando espacios adecuados para ello, cuidando el lenguaje, la empatía, el respeto mutuo, la sana escucha, la alegría, el sentido del humor, la responsabilidad, el optimismo y la actitud positiva frente a los conflictos que se presentan, en sana visión al futuro, recibiendo cada intervención y opinión como la más valiosa.

- Asimismo, el aporte que Valdés brinda a partir de sus estudios sobre familia y desarrollo (Valdés Cuervo, 2007, pág. 98), diferencia una estrategia que ha sido corroborada por otros estudiosos, contribuyendo a enriquecer esta visión frente a los conflictos asociado al desarrollo de acuerdos y el incremento de la confianza.

- Empleo de la Colaboración y el compromiso con alta incidencia en la preocupación por el otro, con una postura de ganancia integrando las necesidades de ambas partes.

Estos aportes son valiosos y de gran eficacia en su aplicación, implican el compromiso de todos los miembros de la familia y los disponen a ser responsables del cambio que se desea. Sin embargo, encontraremos en la propuesta del modelo Harvard -que presentamos a continuación- una herramienta enriquecedora que integra estos aportes y plantea una estructura dinámica, positiva, integradora y personal que fortalece la búsqueda 
de soluciones para estas situaciones de conflicto en las familias.

\section{ESTRATEGIAS DEL MÉTODO DE NEGOCIACIÓN HARVARD}

El modelo Harvard (Correa, 2006) planteado desde la publicación del libro "Si, de acuerdo" por parte del equipo de Negociación de la misma universidad, maneja de manera sencilla la negociación ${ }^{22}$. Este método tiene como plataforma de sustentación -y como su mismo nombre lo señala- una negociación basada en principios $^{23}$. Utilizar esta herramienta reporta ventajas para quién lo conoce y beneficio en cualquier relación de negociación que se establezca, pues basada en principios ambas partes ganarían. A decir de sus mismos autores (Fisher, Ury, \& Patton, 2001, pág. 32), "el método de negociación basado en los principios expuestos y que se centra en los intereses básicos, en las opciones mutuamente satisfactorias y en unos valores justos, da como resultado un acuerdo sabio" 24 . Los cuatro principios (Fisher, Ury, \& Patton, 2001, pág. 27) en los que se basa esta metodología son los siguientes:

1. Personas: Separar las personas del problema.

2. Intereses: Concentrarse en los intereses y no en las posiciones.

3. Opciones: Inventar opciones de mutuo beneficio.

4. Criterios: Insistir en usar criterios objetivos.

\footnotetext{
22 "Una de las cosas más interesantes de la negociación por principios es que se eliminan las luchas por posición que generalmente no tienen ningún sustento y sólo conducen a empantanar las negociaciones y a deteriorar las relaciones" (Trujillo, s.f.).

${ }^{23} \mathrm{El}$ método de negociación según principios consiste en decidir los problemas según sus méritos, en lugar de decidirlos mediante un proceso de regateo centrado en lo que cada parte dice que va o no va a hacer. Sugiere que se busquen ventajas mutuas siempre que sea posible y que cuando haya conflicto de intereses debe insistirse en que el resultado se base en algún criterio justo, independiente de la voluntad de las partes.

(Correa, 2006)

${ }^{24}$ Un acuerdo sabio y prudente puede definirse como uno que aproxima los intereses legítimos de cada una de las partes en el mayor grado posible, resuelve los conflictos de interés de forma justa, es duradero y tiene en consideración los intereses de la comunidad.

(Fisher, Ury, \& Patton, 2001, pág. 22)
}

De aquí es que se derivan los llamados siete elementos (Universidad de Talca, pág. 9) de la negociación (CMI International Group, 1998):

1. Alternativas - son las posibilidades que existen para efectuar un acuerdo entre las partes involucradas, guían el pensamiento y sopesan las alternativas que tiene cada parte. Estas deben estar claras desde el inicio porque ayudan a saber qué debemos o podemos hacer y hasta dónde podemos ceder o cuál es el límite para hacerlo (Trujillo, s.f.).

2. Intereses - son las motivaciones reales que describen lo que quieren y necesitan sus partes. Es el punto de partida exploratorio en donde se definen las posiciones de interés de cada quien para evitar la pérdida de tiempo o el adoptar posturas defensivas que en nada nos benefician (Trujillo, s.f.).

3. Comunicación - necesaria para una buena negociación, busca verificar la real calidad y recursos positivos de una buena comunicación (García Villaluenga, y otros, 2010, págs. 16-21), de manera bilateral, con eficacia y eficiencia. En los casos de búsqueda de acuerdos, la confianza se establece más fácilmente cuando las partes de conocen y lo hacen cara a cara.

4. Relación - es la búsqueda del trabajo conjunto para resolver bien las diferencias. Las relaciones deben ser saludables, armoniosas y sostenibles en el tiempo, más aún cuando se toma conciencia de que "[...] es más fácil lograr acuerdos duraderos cuando se está dentro de una relación de confianza a largo plazo" (Trujillo, s.f.)

5. Opciones - esta es la identificación de toda una gama de posibilidades que tienen las partes para llegar a un acuerdo sin perjudicar a nadie. Este elemento usa activamente la creatividad y la imaginación para generar ideas originales aunque sean diferentes. $Y$ 
aunque parezca sencillo de entender, es importante considerar que "[...] la tentación que debe combatirse es la de pensar que sólo hay una forma de solucionar el problema [...]" (Trujillo, s.f.).

6. Legitimidad - son criterios externos y objetivos desarrollados por otras personas, ajenas a las partes intervinientes, que validen lo justo del acuerdo. Pueden ser leyes, reglamentos o algún principio de aceptación general que como referencia externa vaya más allá de la simple voluntad de los intervinientes.

7. Compromiso - Son los planteamientos viables, verbales o escritos que especificarán lo que las partes harán o dejarán de hacer de común acuerdo. Estos, son resultado de las opciones que fueron estudiadas y evaluadas. Deben de ser prácticos, comprensibles y susceptibles de verificación. Finalmente, al ser viables, requiere entender que requiere de la participación de todos sus miembros.

Es importante en el planteamiento de estrategias, hacer buen uso de estos siete elementos de negociación del método de la Universidad de Harvard (Cane, 2009). Estas son herramientas útiles y prácticas para alcanzar acuerdos satisfactorios en donde todos ganan y nadie pierde, de manera tal que se utilicen sus cuatro ejes principales considerando a las personas, sus intereses, las opciones y los criterios $^{25}$. De estos ejes, se derivan los siete elementos de negociación ya mencionados líneas arriba. Es importante destacar que los siete pasos (CMI International Group, 1998) pueden llevarse uno tras otro o ser manejados independientemente. Indistintamente de la metodología particular que se desee emplear, un paso llevará a otro, si bien, no todos juntos, si varios en conjunto o todos finalmente.

\footnotetext{
${ }^{25}$ Los cuatro elementos de la negociación basada en principios, expuesta por el Proyecto Harvard.
}

Se han mencionado características sobre cada elemento, sin embargo para hacer buen uso de ello, será importante clarificar y acoger también la sana recomendación mediadora de anotar, escribir, registrar y compartir aquello que se ha dispuesto como herramienta de mejora sobre el buen uso de estos elementos de negociación. ¿De qué manera? Acogiendo una herramienta práctica propia de este método, elaborando un cuadro registro donde se indique la problemática en un lado y en el otro la línea de acción a seguir. Para ello será oportuno preguntarnos por cada paso:

- Alternativas - icuáles son las alternativas que tiene cada parte en ese momento? (Trujillo, s.f.) Recordemos que antes de aceptar o rechazar un acuerdo es necesario saber qué otra acción podríamos realizar.

- Intereses - ¿cuáles son mis intereses, cuáles los de los demás? (Trujillo, s.f.). Recordemos que los intereses son susceptibles de alcanzar mientras más se reflexione sobre ellos.

- Comunicación - idirijo mi comunicación considerando el efecto que puede tener en los demás? Recordemos, la comunicación es bilateral y afecta a ambas partes.

- Relación - ésta, imantiene la armonía? (Trujillo, s.f.) Recordemos pensar en construir una relación que facilite, no en entorpecerla.

- Opciones - ¿la posibilidad que tengo, es la única? ¿Qué otra alternativa puedo utilizar? Mientras más opciones podamos ofrecer es más probable que exista una que unifique nuestros intereses.

- Legitimidad - ¿tengo el respaldo suficiente para los criterios que estoy empleando? (Trujillo, s.f.) Recordemos que este es un respaldo para garantizar a todos los participantes la justicia y el respeto en la negociación.

- Compromiso - ¿estoy dispuesto a cumplirlo? Recordemos ser realistas y darle calidad al acuerdo con el establecimiento de promesas que sean factibles y realistas de cumplir. 
Estas estrategias presentadas, basadas en la propuesta del Método Harvard, entre tantos aportes, son de valiosa ayuda para hacer cambios significativamente importantes para su bienestar, integración y superación de dificultades, puesto que proponen acciones progresivas (Maganto, sf.) que se evidencian en la práctica y ejecución de estas estrategias, las cuales conllevan a:

- Pasar progresivamente de actitudes de resentimiento a actitudes de reconciliación, con nosotros mismos, con los demás, con las cosas y las situaciones.

- Pasar progresivamente de actitudes invasoras e impositivas a actitudes acogedoras.

- Pasar progresivamente de actitudes de suspicacia y recelo a actitudes de confianza.

- Pasar de actitudes de intransigencia a actitudes de tolerancia.

- Cambiar de una actitud de rutina y rigidez a una actitud de apertura a los cambios y a la creatividad.

- Cambiar la actitud de aislamiento e incomunicación por una actitud de participación, comunicación y solidaridad.

- Pasar progresivamente de actitudes defensivas a actitudes receptivas y amistosas.

Esta visión de resolución de conflictos, en las familias, es de gran ayuda y soporte, utiliza la creatividad, la participación y el compromiso de todos sus integrantes para aportar soluciones concretas y prevenir sucesos futuros.

La familia es forjadora de valores, en la base donde toda persona crece y se desarrolla, por ello buscar que aprenda a conocer, abordar, enfrentar y hasta agradecer los conflictos es dotarla de medios importantes para su caminar. Desde ella parte todo respeto hacia la persona humana. Su naturaleza y vocación, los derechos que se le confieren y los deberes que asume, hacen que sea digna y que brinde soporte a sus integrantes, sobretodo en su formación integral, buscando su mayor bien, su desenvolvimiento en la sociedad, en defensa de la vida y en el respeto de la dignidad de cada persona y buscando -por esta importancia que tiene para la sociedad y en su función de guía, formación y soporte afectivo como emocional- generar la mayor armonía en cada familia y en consecuencia para cada uno de sus miembros.

\section{Conclusiones}

Se reconoce la importancia de los aportes brindados desde la teoría sistémica, orientados a ser aplicados en diversas áreas del entorno familiar: dentro de la familia, entre padres e hijos, en la mediación, como aporte a Instituciones educativas y en la práctica profesional.

Se han identificado los diversos conflictos que son percibidos en las familias a partir de los resultados de investigaciones similares y afines $y$ contrastadas con la información obtenida en los diálogos con las familias de estudiantes de una Institución educativa.

Se han diferenciado las diversas estrategias que se orientan a las familias para abordar las situaciones de conflicto que se presentan al interior de las familias, las mismas que orientan las acciones a considerar referidas al mejor manejo del conflicto en el hogar.

Se han especificado estrategias a propósito del uso de los elementos de negociación Harvard, considerando las particularidades de cada familia y que van dirigidas a entender básicamente que las familias no son iguales y que para abordar medidas preventivas es necesario conocer sus individualidades.

Es nuestro anhelo el haber contribuido con el tema tratado, en el fortalecimiento de las familias en el dialogo y la mediación para afrontar los conflictos, considerando la importancia y respeto de la persona humana, la búsqueda del bien común, la justicia y la paz 
social, así como la armonía en la familia criterios éticos de esta investigación- por medio de la revisión bibliográfica realizada y la exposición de las estrategias de resolución de conflictos, bajo los criterios del método Harvard de negociación, el cual al ser un método actual, puede motivar a mirar con esperanza a las familias, como lo propone la Teoría sistémica familiar y como esperamos sea enriquecida a partir de estas luces. Es así como consideramos a la familia, eje de la vida, desarrollo y estabilidad de la persona humana, llamada a crecer en comunidad, llamada a servir, llamada a ser sembradora de esperanza y de vida en el amor, el bien y el respeto.

\section{Bibliografía}

Alonso Struyck, P. (2004). Discrepancia entre padres e hijos en la percepción del funcionamiento familiar y desarrollo de la autonomía adolescente. (Tesis Doctoral). Universitat de València.

Arenas Ayllón, S. C. (2009). Relación entre la funcionalidad familiar y la depresión en adolescentes. (Tesis para obtención de Licenciatura). Universidad Nacional Mayor de San Marcos.

Bernedo Muñoz, I., Fuentes Rebollo, M., \& Fernandez Molina, M. (2005). Percepción del grado de conflicto en familias adoptivas y no adoptivas. Psicothema, $17(3)$.

Cane, M. (2009). Ser gerente. Recuperado el 2 de febrero de 2016, de Los siete elementos de negociación de Harvard: http://www.sergerente.net/los-sieteelementos-de-negociacion-de-harvard.

CMI International Group. (1998). Workshop de negociación. Cambridge.

Correa, E. (2006). Gestiopolis. Recuperado el 16 de abril de 2016, de Método Harvard de negociación:

https://www.gestiopolis.com/metodoharvard-de-negociacion/

Espinal, I., Gimeno Collado, A., \& González Sala, F. (2004). El enfoque sistémico en los estudios sobre la familia. Recuperado el 22 de enero de 2015, de Universidad Autónoma de Santo Domingo: http://www.uv.es/jugar2/ Enfoque\%20Sistemico.pdf

Feixas I Viaplana, G., Muñoz Cano, D., Compañ Felipe, V., \& Montesano del Campo, A. (setiembre de 2012). El modelo sistémico en la intervención familiar. Recuperado el 29 de mayo de 2015, de http://diposit.ub.edu/dspace/bitstream/24 45/31584/6/Modelo_Sistemico_Enero201 $6 . p d f$

Fisher, R., Ury, W., \& Patton, B. (2001). Obtenga el sí. El arte de negociar sin ceder. España: Gestión 2000.

Fuquen Alvarado, M. E. (2003). Los conflictos y las formas alternativas de resolución. Tabula Rasa. Recuperado el 20 de julio de 2015, de http://revistatabularasa.org/numero1/Mfuquen.pdf

García Villaluenga, L., Bolaños Cartujo, I., Garrigós Tembleque, S., Gómez Gómez, F., Hierro Requena, M., \& Tejedor Ureta, M. (2010). "¿Cómo resolver conflictos familiares? Madrid: Dirección General de Familia, Comunidad de Madrid. Obtenido de

https://www.ucm.es/data/cont/media/ww w/pag-

41339/COMORESOLVERCONFLICTOSFAMI LIARES.pdf

García Villaluenga, L., Bolaños Cartujo, I., Hierro Requena, M., Garrigós Tembleque, S., Del Hoyo Alonso-Martínez, P., Dorado Barbé, 
A., \& Merino Ortiz, C. (2010). La familia dialoga y llega a acuerdos: La mediación familiar. Madrid: Dirección General de Familia, Comunidad de Madrid. Obtenido de

https://www.ucm.es/data/cont/media/ww $\mathrm{w} /$ pag-

41339/lafamiliadialogayllegaaacuerdos.p $\mathrm{df}$

García, I., Sánchez, A., \& Robles, F. (2009). Factores asociados a la depresión en adolescentes: Rendimiento escolar y dinámica familiar. Anales de psicología, 25(2), 227-240.

Gonzales Gallegos, J. (2007). La familia como sistema. Revista paceña de medicina familiar, 4(6). Recuperado el 10 de enero de 2016, de http://es.calameo.com/books/0031923744 a6ff78f4730

Maganto, M. C. (s.f.). La familia desde el punto de vista sistémico y evolutivo. Recuperado el 25 de febrero de 2016, de http://www.sc.ehu.es/ptwmamac/Capi_lib ro/39c.pdf

Matalinares, M., Arenas, C., Sotelo, L., Díaz, G., Dioses, A., Yaringaño, J., . . . Tipacti, R. (s.f.). Clima familiar y agresividad en estudiantes de secundaria de lima metropolitana. IIPSI Facultad de Psicología UNMSM, 13(1-2010), 109-128.

Motrico, E., Fuentes, M. J., \& Bersabé , R. M. (2001). Discrepancias en la percepción de los conflictos entre padres e hijos/as a lo largo de la adolescencia. Anales de Psicología, 17(1).

Ortiz Granja, D. (2008). La Terapia familiar sistémica. (U. P. Salesiana, Ed.) Quito, Ecuador: Ediciones Abya-Yala.
Papa Francisco. (2016). Exhortación apostólica sobre la familia. La alegría del amor. Asociación católica Evangelización siempre.

Real Academia de la lengua española. (s.f.). Diccionario de la Lengua Española. Recuperado el 20 de julio de 2015, de www.dle.rae.es

Rodríguez Estrada, M. (1989). Manejo de Conflictos. México: El Manual Moderno.

Trujillo, J. (s.f.). Gestiopolis. Recuperado el 20 de abril de 2016, de Negociación personal y el método de Harvard: https://www.gestiopolis.com/negociacion -personal-y-el-metodo-harvard/

UNICEF. ( 2003). "Nuevas formas de familia, perspectivas nacionales $e$ internacionales". Obtenido de Fondo de las Naciones Unidas para la Infancia. Uruguay:

https://www.academia.edu/37542463/NU EVAS_FORMAS_DE_FAMILIA_PERSPECTIV AS_NACIONALES_E_INTERNACIONALES

Universidad de Talca. (s.f.). El conflicto y su resolución. Recuperado el 16 de abril de 2015, de Modelo de negociación de Harvard:

http://studylib.es/doc/141252/negociaci\% C3\%B3n-bajo-el-modelo-de-harvard

Valdés Cuervo, A. (2007). Familia y desarrollo. Intervenciones en terapia familiar. México: Manual Moderno. 